\title{
¿Por qué se le tiene miedo al dentista? \\ Estudio descriptivo de la posición de los pacientes de la Sanidad Pública en relación a diferentes factores subyacentes a los miedos dentales
}

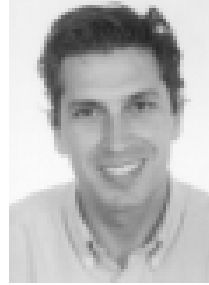

Márquez-Rodríguez Juan Antonio

\section{Why are people afraid of the dentist?}

Márquez-Rodríguez, J uan Antonio*
Navarro- Lizaranzu, Ma: Cruz**
Cruz-Rodríguez, Danie
Gil-Flores, J avier*****

* Odontólogo de Atención Primaria. Distrito Sanitario Huelva-Costa.

** Médico de Medicina Familiar y Comunitaria. Distrito Sanitario HuelvaCosta.

*** Odontólogo de Atención Primaria. Distrito Sanitario Sevilla Sur.

**** Profesor Titular del Área de Métodos de Investigación y Diagnóstico en Educación. Universidad de Sevilla.
Resumen: Introducción: el objetivo del presente artículo es describir la actitud de los pacientes en relación a diferentes factores subyacentes a los miedos dentales. Método: se realiza una encuesta con una muestra de 399 sujetos que acudieron a la consulta de Odontología del Centro de Salud de Lepe (Huelva). Se utilizó un cuestionario con 19 ítemes. El tratamiento estadístico se desarrolló con el paquete de programas SPSS, versión 9.0 (SPSS Inc., Chicago, III, 1999). Resultados: al analizar la estructura factorial de la escala de miedos dentales se encontraron cuatro factores resultantes que se corresponderían con otras tantas dimensiones presentes. El principal temor de los pacientes se centra en las actuaciones terapéuticas que generan o posibilitan daño físico, mientras que los aspectos no relacionados con la profesión les generan menos miedo. Conclusiones: en los miedos al dentista, se identifican cuatro dimensiones o aspectos que podrían diferenciarse y que constituirían respuestas a la pregunta ¿ por qué se le tiene miedo al dentista? Dos de estas dimensiones generan un bajo grado de temor, son las denominadas como aspectos no profesionales y aspectos inherentes al tratamiento. Sin embargo, las otras dos dimensiones sí provocan temor en cierta medida y son las llamadas trato humano, y mala praxis profesional.

Palabras clave: Miedo dental, Ansiedad dental, Sanidad pública.

Abstract: Introduction: the aim of the current article is to describe the patient's attitude regarding several factors hidden in dental fears. Patients and methods: An survey has been performed among 399 patients who visited the Dental department of the Public Health Center localized in Lepe (Huelva, Spain). The questionnaire used included 19 items. The statistical data processing was carried out with the help of the specific software SPSS, version 9.0 (SPSS Inc., Chicago, III, 1999). Results: Analysing the factorial composition of dental fears scale, we detected four factors. The main factor is related to therapeutic actions that may cause physical damage, while factors not related to the professional field generate less fear. Conclusion: among dental fears, it has been possible to differentiate four aspects or dimensions that could represent answers to the question about why people are afraid of the dentist. Two of these dimensions, which generate a low level fear, are the so-called non-professional aspects and treatment inherent aspects. However, the other two dimensions do provoke a certain degree of fear and consist in factors related to the treatment received at a personal level and professional malpractice.

Key words: Dental fears, Dental anxiety, Public health system.

\begin{tabular}{ccc}
\hline Fecha recepción & Fecha última revisión & Fecha aceptación \\
$06-10-2003$ & $19-12-2003$ & $27-02-2004$
\end{tabular}

BIBUD [1138-123X (2004)9:2; marzo-abril 125-256]

Márquez-Rodríguez J A, Navarro-Lizaranzu MC, Cruz-Rodríguez D, Gil-Flores J . ¿Por qué se le tiene miedo al dentista?. RCOE 2004;9(2):165-174.
Juan Antonio Márquez Rodríguez

Plaza de Altisidora, 1 - 3o C

21007 - Huelva

E-mail: juanto@ya.com 


\section{Introducción}

El miedo y la ansiedad son señalados por muchos pacientes como un motivo para no acudir de forma regular al dentista. Esta circunstancia dificulta la atención odontológica de un gran número de pacientes, e incluso puede llegar a impedir el tratamiento dental de algunos individuos $\mathrm{s}^{1,2^{* *}, 3^{*}}$. Existen múltiples publicaciones sobre este asunto; casi todas ellas enuncian que el temor al dentista es más numeroso de lo que pudiera parecer, y por tanto, no es una situación excepcional.

La ansiedad y los miedos dentales, desde la perspectiva conductual, se deben considerar como patrones multidimensionales y aprendidos de comportamiento que resultan desadaptativos en relación al tratamiento odontológico ${ }^{3 *}$. En el desarrollo de estas actitudes intervienen múltiples causas y factores, por lo que el origen de la ansiedad y el miedo a la atención dental se postula como multifactorial por la mayoría de los autores. Los factores señalados son variados, pero los más relacionados son: la conducta miedosa aprendida del modelo familiar o su entorno ${ }^{2 * *}$; el sexo femenino parece experimentar mayor miedo y ansiedad, aunque existen estudios que no encuentran diferencias significativas entre hombres y mujeres $^{2 * * 4}$; los niños y jóvenes son más ansiosos y miedosos ${ }^{2 * *, 5}$; las experiencias desagradables previas, especialmente durante la infancia ${ }^{2 * *, 6}$; existen variaciones por etnias, aunque esto es difícil de valorar por las diferencias entre culturas y costumbres ${ }^{2 *, 7}$; los factores socioeconómicos también parecen influir, sobre todo los facto- res sociales (familias rotas, drogadicción, problemas de educación, etc. ${ }^{2 * *}$; las personas con malos hábitos higiénicos suelen ser más ansiosas, al igual que aquellos que tienen un mal estado bucodental ${ }^{2 * *}$; las personas con discapacidad psíquica experimentan más miedo a los tratamientos odontológicos por su falta de comprensión ${ }^{2 * *}$; los pacientes que padecen enfermedades crónicas y graves suelen presentar menos ansiedad porque consideran un problema menor su estado bucal ${ }^{2 * *}$; por último, un tiempo de espera prolongado antes de la atención, un número excesivo de pacientes en la sala de espera porque al individuo le parece que el tratamiento se realiza con prisas, y sesiones de tratamiento de larga duración parecen aumentar el nivel de ansiedad $^{2 * * 8}$.

El concepto más universalmente aceptado de ansiedad se refiere a un complejo patrón de conducta asociado a una activación fisiológica, que ocurre en respuesta a estímulos internos (cognitivos y somáticos) y externos (ambientales), que pueden aparecer antes y durante el proceder odontológico en los pacientes sin que lo identifiquemos ${ }^{2 * *}$, mientras que el miedo se puede definir como una perturbación angustiosa del ánimo ante una situación peligrosa real o imaginaria. Existen tres elementos diferenciadores entre la ansiedad y los miedos dentales. El principal elemento reside en la dimensión temporal entre la conducta y el ambiente propio del tratamiento dental. La ansiedad dental sigue un patrón conductual anticipatorio, evocado por una situación preaversiva; es decir, se presenta de manera anticipada al trata- miento. Los miedos dentales, por el contrario, revisten un carácter contemporáneo al tratamiento, tienen una relación de inmediatez temporal con el tratamiento odontológico. El segundo elemento de diferenciación es la generalización de la respuesta: la ansiedad dental se da como un patrón de respuesta generalizado, evocado por el contexto global del tratamiento, mientras que los miedos dentales se caracterizan como patrones discriminativos, controlados por estímulos específicos durante el tratamiento. Finalmente, los elementos operantes-motores por parte del paciente en la ansiedad son de evitación del tratamiento, mientras que los que conllevan los miedos dentales son de escape del tratamiento, que se traducen en comportamiento perturbador u obstaculizante durante la intervención $3^{3 *}$.

Se puede afirmar que la persona con miedo y/o ansiedad ante la situación terapéutica dental provoca para sí misma y para el dentista unas consecuencias que alteran el correcto orden y desarrollo de una cita y, si se da el caso, de un plan de tratamiento ${ }^{2 * *}$.

En el presente estudio pretendemos describir los miedos dentales expresados por los pacientes que acuden al dentista, identificando para ello los factores o dimensiones que forman parte del concepto de miedo dental.

\section{Pacientes y método}

El estudio, por su finalidad descriptiva, se ha basado metodológicamente en un diseño de investigación mediante procedimiento de encuesta. 


\section{Población y muestra}

La población considerada en el estudio está constituida por los sujetos comprendidos entre 10 y 80 años de edad, de ambos sexos, que acuden a la consulta de odontología de la sanidad pública en la Zona Básica de Salud de Lepe (Huelva). De cara a facilitar la recogida de datos, se ha seguido un procedimiento de muestreo incidental, de tal manera que se seleccionaron aquellos sujetos que durante los meses de marzo, abril y mayo del año 2001 acudieron a la consulta de odontología del Centro de Salud de esta localidad y quisieron participar en el estudio. Cabe pensar que es una muestra representativa del colectivo atendido durante este período en esta consulta.

En cuanto al tamaño de la muestra, ésta ha ascendido a un total de 399 sujetos. En el supuesto de un muestreo aleatorio, este tamaño muestral permite trabajar con una confianza del $95,5 \%$ y un error del $5 \%$ para $p=q$. El tamaño alcanzado por la muestra posibilita la realización de inferencias sobre la población con unos márgenes de precisión y confianza aceptables.

\section{Material para la recogida de datos}

Se ha utilizado como base para esta investigación el cuestionario de miedos dentales ${ }^{3 *}$, que admite puntuaciones en un rango de 0-100, y ha obtenido índices de confiabilidad de alfa de Cronbach $=0,92$. Además de por su fiabilidad, era apropiado para el estudio por su comprensión y la uniformidad de su código. Este cuestionario se compone de 20 ítemes referidos a actividades y experiencias

\section{Anexo l': Cuestionario utilizado en el estudio sobre miedos dentales}

Instrucciones: las frases de este cuestionario se refieren a actividades y experiencias relacionadas con la atención dental que podrían producirle miedo $\mathrm{u}$ otros sentimientos desagradables.

Lea cada frase y piense cuánto le molesta en la actualidad, luego rodee con un círculo el valor correspondiente al grado de temor que le causa cada una de las frases, de acuerdo con los siguientes códigos. Procure no dejar ninguna sin contestar.

\section{Código de miedo experimentado \\ 0 ninguno en absoluto \\ 1 un poco \\ 2 ligeramente \\ 3 moderadamente \\ 4 mucho \\ 5 muchísimo}

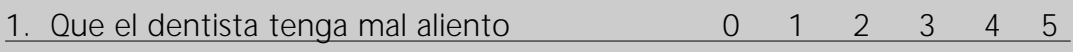

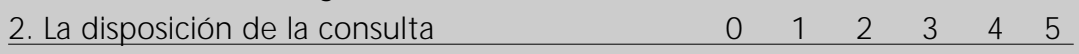

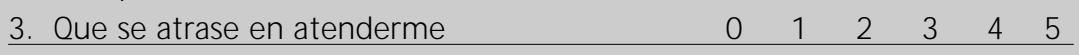

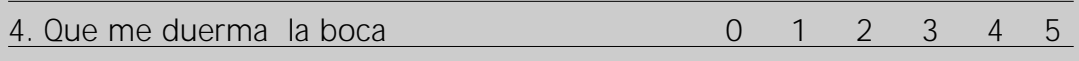

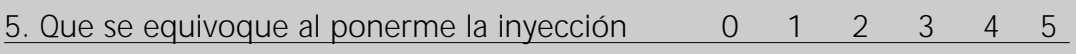

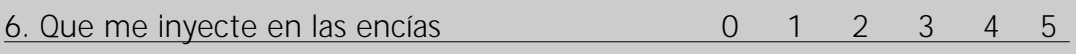

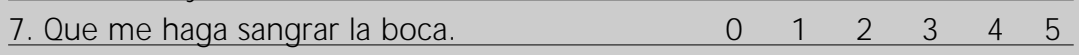

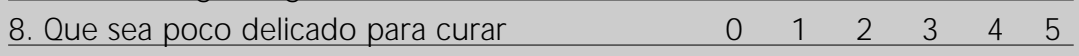

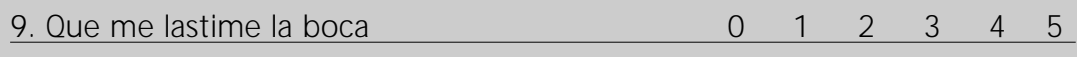

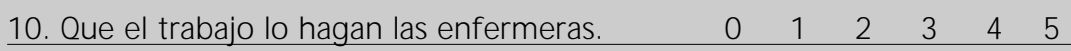

11. Que me rompa los labios con algún instrumento

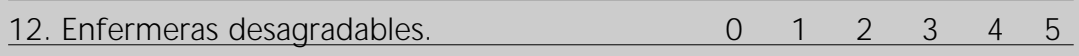

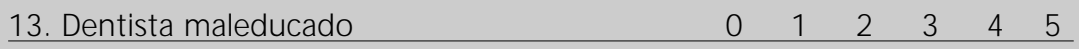

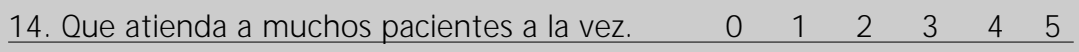

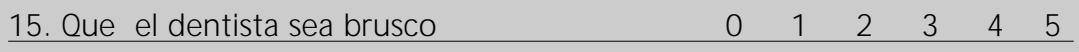

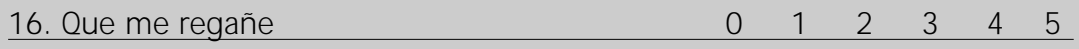

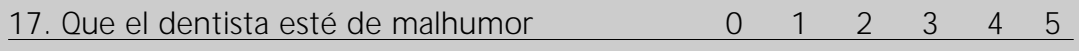

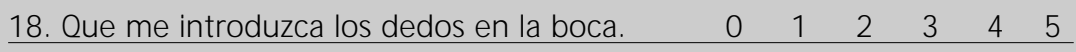

19. Que me pueda transmitir infecciones con los instrumentos

$\begin{array}{llllll}0 & 1 & 2 & 3 & 4 & 5\end{array}$

relacionadas con la situación dental que pueden producir miedo $u$ otros sentimientos desagradables. Se han suprimido los dos primeros ítemes (1. Que me coloquen el abrebocas, 2. Las revistas viejas), por no ser situaciones 


\begin{tabular}{|c|c|c|c|c|c|c|c|c|}
\hline \multicolumn{9}{|c|}{$\begin{array}{l}\text { Tabla l: Porcentajes del grado de miedo, medias y desviacio } \\
\text { para los ítemes de la escala de miedos dentales }\end{array}$} \\
\hline & $\mathbf{n}$ & $\begin{array}{c}\text { \%Ningún miedo } \\
\text { en absoluto }\end{array}$ & $\begin{array}{l}\% \text { Un poco } \\
\text { de miedo }\end{array}$ & $\begin{array}{c}\text { \%Miedo } \\
\text { ligero }\end{array}$ & $\begin{array}{r}\% \text { Miedo } \\
\text { moderado } \\
\end{array}$ & $\begin{array}{c}\% \text { Mucho } \\
\text { miedo }\end{array}$ & $\begin{array}{l}\text { \%uchísimo } \\
\text { miedo }\end{array}$ & $\begin{array}{l}10 \text { Media } \\
\text { (Desv. Típ.) }\end{array}$ \\
\hline $\begin{array}{l}\text { Que me pueda transmitir infecciones } \\
\text { con los instrumentos }\end{array}$ & 398 & 13,8 & 5,7 & 5,2 & 6,0 & 12,0 & 57,0 & $3,6(1,8)$ \\
\hline $\begin{array}{l}\text { Que me rompa los labios con algún } \\
\text { instrumento }\end{array}$ & 398 & 13,0 & 8,7 & 4,7 & 8,0 & 17,0 & 48,2 & $3,5(1,8)$ \\
\hline Que el dentista sea brusco & 398 & 13,5 & 6,2 & 7,5 & 10,3 & 22,8 & 39,4 & $3,4(1,7)$ \\
\hline Que me lastime la boca & 398 & 10,5 & 9,5 & 8,0 & 12,3 & 25,6 & 33,9 & $3,3(1,7)$ \\
\hline Que sea poco delicado para curar & 394 & 11,6 & 12,1 & 8,8 & 12,9 & 20,3 & 34,0 & $3,2(1,7)$ \\
\hline Dentista maleducado & 396 & 18,6 & 8,0 & 5,5 & 10,8 & 21,2 & 35,6 & $3,1(1,9)$ \\
\hline $\begin{array}{l}\text { Que se equivoque al ponerme } \\
\text { la inyección }\end{array}$ & 398 & 17,0 & 14,3 & 7,2 & 6,7 & 20,3 & 34,1 & $3,0(1,9)$ \\
\hline Que el dentista esté de mal humor & 398 & 19,8 & 9,8 & 9,0 & 14,5 & 16,3 & 30,4 & $2,8(1,9)$ \\
\hline Enfermeras desagradables & 398 & 20,8 & 8,7 & 8,2 & 15,0 & 17,3 & 29,6 & $2,8(1,9)$ \\
\hline Que atienda a muchos pacientes a la vez & 396 & 21,4 & 9,0 & 9,0 & 18,6 & 15,1 & 26,5 & $2,7(1,8)$ \\
\hline Que el trabajo lo hagan las enfermeras & 394 & 23,8 & 13,7 & 7,1 & 15,7 & 15,9 & 23,6 & $2,5(1,9)$ \\
\hline Que me regañe & 399 & 25,5 & 15,0 & 8,5 & 16,7 & 13,2 & 20,8 & $2,4(1,9)$ \\
\hline Que me inyecte en las encías & 395 & 27,0 & 17,9 & 10,6 & 12,9 & 12,1 & 19,2 & $2,2(1,8)$ \\
\hline Que me haga sangrar la boca & 398 & 30,6 & 16,5 & 9,8 & 14,3 & 15,0 & 13,5 & $2,0(1,8)$ \\
\hline Que me introduzca los dedos en la boca & 396 & 34,3 & 14,6 & 8,8 & 14,9 & 12,3 & 14,9 & $2,0(1,8)$ \\
\hline Que se atrase en atenderme & 396 & 30,0 & 26,0 & 7,3 & 13,6 & 11,8 & 11,1 & $1,8(1,7)$ \\
\hline La disposición de la consulta & 394 & 34,7 & 17,2 & 13,7 & 13,2 & 11,4 & 9,6 & $1,7(1,7)$ \\
\hline Que el dentista tenga mal aliento & 395 & 46,8 & 10,6 & 5,8 & 9,8 & 9,8 & 16,9 & $1,7(1,9)$ \\
\hline Que me duerma la boca & 393 & 50,6 & 16,2 & 7,1 & 10,4 & 5,6 & 9,9 & $1,3(1,7)$ \\
\hline n válido (según lista) & 365 & & & & & & & \\
\hline
\end{tabular}

que se puedan dar en la consulta donde se desarrolló la encuesta.

Antes de comenzar la encuesta, durante el mes de febrero de 2001, se llevó a cabo un estudio piloto que consistió en aplicar el instrumento que se utilizaría en el estudio a un conjunto reducido de 20 sujetos de la misma población. A partir de este estudio se pretendió determinar la validez del cuestionario, entendida como su adecuación de contenidos y forma para las características de los sujetos desti- natarios, lo que se comprobó tras este pilotaje. Tan sólo se añadió el último ítem (que me pueda transmitir infecciones con los instrumentos) tras el comentario de uno de los participantes en el estudio piloto, y que probablemente sería un supuesto que podría causar miedo. Por ello, el cuestionario de miedos dentales utilizado ha quedado finalmente con diecinueve ítemes. Las respuestas posibles adoptan el formato de una escala de seis puntos (valores de 0 a 5 ) que van des- de «ningún miedo» hasta «muchísimo miedo» experimentado ante las situaciones que se describen (Anexo 1).

\section{Procedimientos de análisis}

La información recogida mediante el cuestionario fue codificada numéricamente y almacenada en una matriz de datos. El tratamiento estadístico de esta matriz numérica se realizó utilizando el paquete de programas SPSS, versión 9.0 (SPSS Inc., Chicago, III, 1999). 
De cara a responder al objetivo eminentemente descriptivo del trabajo, se ha recurrido a la descripción de los datos a partir de la construcción de distribuciones de frecuencias (absolutas y relativas expresadas como porcentajes) para los resultados en cada ítem, a las que se han unido el cálculo de medidas de tendencia central (media) y de dispersión (desviación típica).

Para identificar la estructura factorial de los miedos dentales, se ha recurrido a la técnica del análisis factorial por el método de componentes principales, seguido de una rotación de factores con el método varimax. La idoneidad de los datos para la aplicación de este análisis se ha contrastado a partir de diferentes medidas (KMO, test de Barlett).

El examen de los pesos de cada ítem en los factores obtenidos ha permitido agrupar los 19 ítemes de la escala de miedos dentales en un número reducido de dimensiones, contribuyendo a interpretar su significado. Además, para cada uno de los factores se ha calculado la puntuación media alcanzada por los sujetos, obtenida como promedio de las puntuaciones registradas en cada uno de los ítemes que integran el factor. Las puntuaciones factoriales obtenidas por este procedimiento han permitido describir la posición de los pacientes de la odontología pública en relación a las diferentes dimensiones subyacentes al constructo «miedos dentales».

Finalmente, un análisis de la varianza seguido de comparaciones posteriores mediante la prueba de Scheffe, ha servido para valorar la existencia de diferencias significativas entre las puntuaciones medias logradas en los factores.

\section{Resultados}

\section{Descripción de los miedos dentales}

A la hora de describir los datos conseguidos con el cuestionario de miedos dentales se ha encontrado que algunas de las situaciones propuestas en los ítemes generan bastante miedo, mientras que otras lo producen levemente. Entre las primeras sobresale de manera llamativa la posibilidad de recibir la transmisión de infecciones con los instrumentos, con un $57,0 \%$ de los sujetos que perciben la situación como muchísimo miedo, seguida del supuesto que me rompan los labios con algún instrumento, con un $48,2 \%$ que también sienten muchísimo miedo ante tal posibilidad. Por el contrario los ítemes que provocan menos miedo son que me duerma la boca que produce un $50,6 \%$ y que el dentista tenga mal aliento con un $46,8 \%$ de ningún miedo en absoluto en ambos casos, entre los encuestados de la muestra (tabla 1).

Además se ha hallado la media aritmética para cada ítem, y con los valores conseguidos se consideró que aquellas situaciones con una media inferior a 2,5 producen un escaso o nulo miedo y las que tienen un valor superior a 2,5 provocan miedo en grado medio o elevado. De esta manera, aparecen un total de once situaciones o experiencias que generan un cierto grado de miedo y otras ocho que dan lugar a poco o ningún miedo entre los individuos integrantes de la muestra. También en esta división figuran los mismos ítemes de la anterior distribución de porcentajes como los más sobresalientes tanto para la situación de miedo, como para la situación de escaso o nulo miedo (tabla 1 ).

\section{Identificación de los fac- tores en la Escala de Mie- dos Dentales}

Un paso previo a la extracción de factores mediante el análisis factorial ha sido el estudio de la matriz de correlaciones, a fin de valorar si el grado de correlación entre las variables es suficiente para plantear la aplicación de esta técnica de análisis. El test de esfericidad de Barlett arrojó un valor de 3711,53, significativo con una confianza superior al 99,99\% El valor obtenido en el test de Kaiser-Meyer-Olkin de adecuación de muestreo fue de 0,923 , que puede calificarse como «maravillosa» de cara a la aplicación del análisis factorial, según la escala elaborada por Kaiser ${ }^{9}$. Ambas pruebas confirman por tanto la idoneidad de los datos para llevar a cabo el análisis factorial con este grupo de variables.

\section{Estructura factorial de la Escala de Miedos Dentales}

La solución encontrada para identificar la estructura factorial de la escala los miedos dentales ofrece cuatro factores, que explican el $65,0 \%$ de la varianza total (tabla 2). Un examen de los ítemes que presentan pesos factoriales altos en cada factor (tabla 3), permite la identificación del significado de los mismos. Los ítemes 10, 18 y 19 presentan pesos altos en más de un factor, estando más próximos por su contenido semántico a factores en los que no registran las cargas factoriales más altas. Estos ítemes han sido eliminados por considerarlos variables factorialmente complejas que dificultarían la interpretación de los factores. 


\begin{tabular}{|c|c|c|c|c|c|c|}
\hline \multicolumn{7}{|c|}{$\begin{array}{c}\text { Tabla 2: Varianza explicada por los componentes } \\
\text { tras la rotación varimax }\end{array}$} \\
\hline \multirow[b]{2}{*}{ Componente } & \multirow[b]{2}{*}{ Autovalor } & \multirow[b]{2}{*}{ varianza } & \multirow[b]{2}{*}{$\begin{array}{c}\text { \%de la } \\
\text { acumulado }\end{array}$} & \multicolumn{3}{|c|}{$\begin{array}{l}\text { Suma de las saturaciones } \\
\text { al cuadrado de la rotación }\end{array}$} \\
\hline & & & & $\begin{array}{c}\% \\
\text { Total }\end{array}$ & $\begin{array}{l}\text { \%de la } \\
\text { varianza }\end{array}$ & $\begin{array}{c}\% \\
\text { acumulade }\end{array}$ \\
\hline 1 & 8,045 & 42,3 & 42,3 & 4,5 & 23,8 & 23,8 \\
\hline 2 & 1,979 & 10,4 & 52,7 & 3,7 & 19,7 & 43,5 \\
\hline 3 & 1,236 & 6,5 & 59,2 & 2,0 & 10,7 & 54,3 \\
\hline 4 & 1,099 & 5,7 & 65,0 & 2,0 & 10,7 & 65,0 \\
\hline 5 &, 785 & 4,1 & 69,1 & & & \\
\hline 6 & ,709 & 3,7 & 72,9 & & & \\
\hline 7 & ,668 & 3,5 & 76,4 & & & \\
\hline 8 & ,613 & 3,2 & 79,6 & & & \\
\hline 9 &, 506 & 2,6 & 82,3 & & & \\
\hline 10 &, 469 & 2,4 & 84,7 & & & \\
\hline 11 &, 425 & 2,2 & 87,0 & & & \\
\hline 12 & ,412 & 2,1 & 89,1 & & & \\
\hline 13 & ,388 & 2,0 & 91,2 & & & \\
\hline 14 & ,383 & 2,0 & 93,2 & & & \\
\hline 15 &, 335 & 1,7 & 95,0 & & & \\
\hline 16 & ,289 & 1,5 & 96,5 & & & \\
\hline 17 & ,257 & 1,3 & 97,8 & & & \\
\hline 18 & ,213 & 1,1 & 99,0 & & & \\
\hline 19 & , 188 &, 9 & 100,0 & & & \\
\hline
\end{tabular}

Los cuatro factores resultantes se corresponderían con otras tantas dimensiones presentes en la estructura de los miedos dentales. Las dimensiones, junto con los ítemes incluidos en cada una de ellas y la denominación que en función del contenido de éstos se le ha dado a cada dimensión son como se presentan a continuación.

Dimensión 1: Trato humano al paciente.

Ítem 17. Que el dentista esté de mal humor.

Ítem 16. Que me regañe.

Ítem 13. Dentista maleducado.

Ítem 15. Que el dentista sea brusco.

Ítem 12. Enfermeras desagradables.

Ítem 14. Que atienda a muchos pacientes a la vez.
Dimensión 2: Mala praxis profesional. Ítem 11. Que me rompa los labios con algún instrumento.

Ítem 9. Que me lastime la boca.

Ítem 5. Que se equivoque al ponerme la inyección.

Ítem 8. Que sea poco delicado para curar.

Ítem 7. Que me haga sangrar la boca. Dimensión 3: Aspectos inherentes al tratamiento.

Ítem 4. Que me duerma la boca. Ítem 6. Que me inyecten en las encías.

Dimensión 4: Aspectos no profesionales.

Ítem 2. La disposición de la consulta.

Ítem 1. Que el dentista tenga mal aliento.

Ítem 3. Que se atrase en atenderme.
Fiabilidad de la medición de los factores de la Escala de Miedos Dentales

Para establecer la fiabilidad de la escala de miedos dentales, se ha recurrido al cálculo del coeficiente alfa de Cronbach, que mide la consistencia interna del instrumento. Este cálculo se han repetido con cada una de las subescalas (conjuntos de ítemes incluidos en cada factor) que se han logrado identificar con el análisis de componentes principales. El examen de los valores obtenidos para estos coeficientes (tabla 4), permite comprobar la elevada fiabilidad de los instrumentos de medida empleados.

El valor para la escala considerada globalmente es de 0,92, y se mantiene en valores altos en las subescalas, a pesar de la reducción en el número de ítemes. Téngase en cuenta al respecto, el efecto que tiene la reducción del tamaño del instrumento sobre la fiabilidad del mismo. En consecuencia, se pueden considerar fiables las medidas obtenidas con la escala de miedos dentales y también por cada una de las subescalas consideradas.

\section{Descripción de los facto- res de la Escala de Miedos Dentales}

Si se atiende a los resultados obtenidos en cada dimensión de la Escala (tabla 5), se pueden describir los miedos dentales de los pacientes de la consulta odontológica en la sanidad pública, basándose en los factores subyacentes.

El principal temor de los pacientes se centra en lo que se ha denominado una mala praxis profesional, dimensión en la que se alcanza una media de 3,0; es decir, los pacientes te- 


\begin{tabular}{|c|c|c|c|}
\hline \multicolumn{4}{|c|}{$\begin{array}{l}\text { Tabla 3: Saturaciones de los ítemes en cada uno de los factores } \\
\text { para la solución rotada }\end{array}$} \\
\hline Componente & 2 & 3 & 4 \\
\hline Que el dentista esté de mal humor & & & \\
\hline Que me regañe & & & \\
\hline Dentista maleducado & & & \\
\hline Que el dentista sea brusco & 361 & & \\
\hline Enfermeras desagradables & 413 & & \\
\hline $\begin{array}{l}\text { Que me pueda transmitir infecciones } \\
\text { con los instrumentos }\end{array}$ & ,456 & & \\
\hline Que atienda a muchos pacientes a la vez & & & 345 \\
\hline Que me introduzca los dedos en la boca & & ,458 & \\
\hline Que me rompa los labios con algún instrumento ,366 & 777 & & \\
\hline Que me lastime la boca & ,772 & & \\
\hline Que se equivoque al ponerme la inyección &, 722 & & \\
\hline Que sea poco delicado para curar & ,715 & & \\
\hline Que me haga sangrar la boca & ,597 & ,490 & \\
\hline Que el trabajo lo hagan las enfermeras & ,469 & & \\
\hline Que me duerma la boca & & ,833 & \\
\hline Que me inyecte en las encías & & ,743 & \\
\hline La disposición de la consulta & & & 838 \\
\hline Que el dentista tenga mal aliento & & & 776 \\
\hline Que se atrase en atenderme & & 406 & 433 \\
\hline $\begin{array}{l}\text { Método de extracción: Análisis de componentes principa } \\
\text { Método de rotación: Normalización Varimax con Kaiser. }\end{array}$ & & & \\
\hline
\end{tabular}

\begin{tabular}{|l}
\hline \multicolumn{4}{|c|}{ Tabla 4: Coeficientes alfa de Cronbach para la escala } \\
$\qquad y$ las cuatro subescalas identificadas
\end{tabular}

men que como consecuencia del tratamiento dental se le produzca algún tipo de daño, tales como la rotura de los labios, las heridas en la boca y el sangrado de la misma, lo cual se vin- cula a la poca delicadeza del dentista en su práctica. También los temores se dirigen hacia los posibles errores que pudiera cometer el odontólogo, por ejemplo al poner la inyección.
Otro de los aspectos que suscitan temor en los pacientes, aunque en menor medida, son los que tienen que ver con el trato humano dispensado al paciente (media de 2,9). Éste expresa el temor ante la posibilidad de ser atendido por un dentista que esté de mal humor, que le riña, que sea maleducado, o que sea brusco. También le afectaría si el personal de enfermería es desagradable, y el supuesto de ser tratado junto a otros pacientes a la vez.

En cambio, puede decirse que no producen temor entre los pacientes otros aspectos que, o bien son inherentes, y por tanto inevitables, al tratamiento dental (media de 1,8) o bien tienen que ver con cuestiones no estrictamente profesionales (media de $1,8)$. En el caso de los aspectos inherentes se encuentra que los pacientes asumen sin mucho temor el hecho de sentir su boca dormida o que le inyecten en sus encías. Los aspectos no relacionados con la profesión también generan escaso miedo; así le afecta menos la disposición de la consulta, que el dentista tenga mal aliento, 0 que le atiendan con retraso.

Para comprobar que cada uno de los factores identificados generan diferente grado de temor en los pacientes, se ha llevado a cabo el contraste de las medias obtenidas para cada dimensión a través de un análisis de la varianza. El estadístico de contraste (F) ha alcanzado el valor 81,29 , que permite rechazar la hipótesis nula de igualdad de medias con una confianza próxima al $100 \%(p=0,000)$. Las comparaciones posteriores mediante la prueba de Scheffe permiten afirmar la existencia de diferencias significativas $(\alpha=0,05)$ entre las medias al- 


\begin{tabular}{|c|c|c|c|}
\hline \multicolumn{4}{|c|}{$\begin{array}{l}\text { Tabla 5: Medias aritméticas del grado de miedo percibido } \\
\text { en cada dimensión }\end{array}$} \\
\hline & $\mathbf{n}$ & Media & Desviación típica \\
\hline Trato humano al paciente & 391 & 2,9 & 1,6 \\
\hline Mala praxis profesional & 390 & 3,0 & 1,5 \\
\hline Aspectos inherentes al tratamiento & 390 & 1,8 & 1,6 \\
\hline Aspectos no profesionales & 389 & 1,8 & 1,4 \\
\hline
\end{tabular}

canzadas en los factores primero y segundo, de una parte, y los factores tercero y cuarto de otra. Es decir, los temores expresados en relación a una mala praxis profesional y al trato que dispensa el dentista al paciente son significativamente superiores a los que se deberían a aspectos inherentes al tratamiento o a aspectos no profesionales.

\section{Discusión}

Al analizar los miedos al dentista, se identifican cuatro dimensiones o aspectos que podrían diferenciarse $y$ que constituirían respuestas al interrogante que se planteaba en el título del trabajo. Dos de estos factores generan un bajo grado de temor, son los denominados como aspectos no profesionales y aspectos inherentes al tratamiento. Sin embargo, los otros dos factores sí provocan temor en cierta medida y son los llamados trato humano, y mala praxis profesional.

Es indudable que la ansiedad y los miedos dentales afectan negativamente a la promoción y la viabilidad del tratamiento odontológico $\mathrm{y}$, por ende, repercuten de forma desfavorable en la salud dental de la población ${ }^{3 *}$.
Sin duda alguna, el primer efecto negativo de los miedos y la ansiedad va a ser la conducta de evitación por parte del paciente. Esta actitud de evasión va a interferir el seguimiento del tratamiento $y$, a veces, es una barrera infranqueable para el acceso a la salud oral de algunos de estos individuos, y ésta es la peor de las consecuencias. Berggren dice que la ansiedad ante la odontología crea su propio círculo vicioso, en el que la evasión del tratamiento dental consigue un deterioro de la dentición que da lugar a sentimientos de culpa, de compromiso y de inferioridad. Con el paso del tiempo este «conflicto social» refuerza la ansiedad y resulta una nueva evasión del tratamiento dental ${ }^{2 *, 3 * 10,11}$. El mal estado bucal, provocado en cierto modo por su conducta de evasión, reduce la autoestima de estos individuos e impide la consecución de una calidad de vida adecuada ${ }^{2 * *}$.

Otro inconveniente es el aumento en los costos operativos y psicológicos como resultado de la conducta perturbadora. Un porcentaje importante de estos individuos presenta mala cooperación durante el tratamiento y en algunos la colaboración es nula, lo que contribuye a hacer más difícil el correcto desarrollo del tratamiento dental, además de disminuir la calidad de los tratamientos ${ }^{3 *}$. Además la ansiedad disminuye el umbral de tolerancia al dolor y aumenta la posibilidad de que estímulos no nocivos sean interpretados por el paciente como dolorosos ${ }^{2 * * 4}$.

Es evidente que la aparición de estas conductas altera la relación profesional-paciente y afecta negativamente al dentista y al personal del gabinete odontológico, lo que desemboca en fatigas y fracasos profesionales, también provocados por tratamientos incompletos. Además estos pacientes recuerdan tratamientos dentales previos desagradables, y adoptan una perspectiva del profesional dentista sesgada ${ }^{2 * *}$.

Algunos autores utilizan el miedo para motivar; sin embargo, se ha visto que el miedo tiene un promedio de vida muy corto, y por tanto, cambios de conducta conseguidos por medio de éste enseguida volverán a modificarse en cuanto desaparezca. En muchas ocasiones, las críticas o llamadas de atención sobre la higiene oral no van a lograr un cambio de actitud en el paciente, sino que incluso éste puede vivirlo como una experiencia traumática y, por tanto, ser un elemento influyente en la aparición de miedo y ansiedad en la consulta dental ${ }^{12}$.

En el presente estudio, las actividades vividas durante el tratamiento que provocan más miedo fueron las actuaciones terapéuticas que generan o posibilitan daño físico al individuo. Esto se corresponde con otros estudios, que manifiestan que el mero hecho de ver, oír o sentir la turbina, la aguja, una herida o sangre ya genera una conducta negativa ${ }^{4,13}$. En este sentido, es importante reseñar que una experiencia traumática o doloro- 
sa, fundamentalmente durante la niñez, es el elemento influyente aceptado y referido de forma más amplia por los pacientes a la hora de argumentar su miedo o ansiedad ${ }^{2 * *}$.

Las circunstancias relacionadas con el trato humano concedido por los profesionales también son referidas por los encuestados como un factor importante en la aparición de miedo en la consulta; de esta manera, algunos autores mencionan que la antipatía a la persona del odontólogo se puede considerar un posible precursor de ansiedad ${ }^{2 *}$, mientras que los aspectos inherentes al tratamiento odontológico y los aspectos no profesionales que lo rodean, habitualmente producen poco miedo. Sin embargo, la angustia que despierta el tratamiento odontológico es de tipo emocional, pues nada de lo que acontece en el tratamiento explica la ansiedad que produce, ni las reacciones que se observan en el paciente ${ }^{4}$, de tal forma, que, a veces, podemos encontrarnos individuos que no hayan experimentado dolor o tratamiento dental traumático y, no obstante, han aprendido por asociación del modelo familiar o su entorno que el dentista y su tratamiento provocan miedo y ansiedad. Es el caso de algunos niños, donde la ansiedad ante la odontología de la madre actúa de manera influyente en la del hijo ${ }^{2 *}$.

Los pacientes tienden a reducir sus niveles de ansiedad y a experimentar menos miedo con la edad y a medida que se visita al dentista periódicamente, siempre que no existan experiencias negativas. A pesar de ello, existe una alta prevalencia de ansiedad y miedo ante una intervención dental y son los profesionales de la salud bucodental los que deben adquirir las habilidades necesarias para su manejo, porque estos pacientes desean controlar su problema ${ }^{3 *}$.

Entre las disciplinas que se dedican a la prevención y tratamiento del miedo dental está la odontología conductual que aplica los principios teóricos, metodológicos y tecnológicos de la psicología conductual al ámbito extenso de la salud dental, pero principalmente a la prevención y tratamiento de la ansiedad dental y de los miedos dentales mediante la aplicación de múltiples técnicas orientadas al cambio conductual ${ }^{3 *}$. La utilización de las benzodiacepinas por diferentes vías de administración (oral, rectal, intranasal, intravenosa) es frecuente y sobradamente conocida en la prevención de estos problemas ${ }^{14-17}$. Otra alternativa es el uso de óxido nitroso que también se muestra eficiente en la prevención de estos sucesos ${ }^{16,18}$. Otros profesionales obtienen una reducción sustancial de la ansiedad y el miedo dental a través de sencillas sesiones de reestructuración cognitiva, modificando las ideas negativas que los sujetos tienen, perdurando en el tiempo la reducción del miedo conseguida ${ }^{17,19-22}$. Incluso existen métodos más singulares para intentar disminuir los inconvenientes que acompañan a esas conductas temerosas; como ejemplo la experiencia de una clínica dental que ambientada con olor a naranja logró reducir la ansiedad y las alteraciones del humor en mujeres ${ }^{23}$.

Cualquier procedimiento puede ser útil si se consigue una disminución del temor dental antes y durante la atención dental, pero también es fundamental evitar las situaciones que conllevan daño al paciente durante el tratamiento y optimizar el trato humano dado por los profesionales de los servicios dentales.

\section{Bibliografía recomendada}

Para profundizar en la lectura de este tema, el/los autor/es considera/an interesantes los artículos que aparecen señalados del siguiente modo: *de interés **de especial interés.

1. Márquez Rodríguez JA. Estudio sobre los conocimientos, comportamientos $y$ actitudes respecto a la salud oral en pacientes de la Sanidad Pública. Trabajo de investigación para la titulación de Experto Universitario en Salud Pública Oral. Vicerrectorado de Tercer Ciclo y Enseñanzas Propias. Universidad de Sevilla. 2001.

\footnotetext{
2**. Aguilera F, Osorio R, Toledano M. Causas, factores $y$ consecuencias implicados en la aparición de ansiedad en la consulta dental. Rev Andal Odontol Estomatol 2002;12:21-4. Este artículo realiza una exhaustiva revisión sobre las causas, factores y consecuencias relacionados con las situaciones temerosas durante la atención odontológica.
}

3*. Navarro C, Ramírez R. Un estudio epidemiológico acerca de la prevalencia de ansiedad $y$ miedos dentales entre la población adulta de la gran área metropolitana de Costa Rica. Psicología Conductual 1996;4:79-95.

Este estudio establece parámetros de prevalencia de ansiedad y miedos dentales, y analiza esta problemática desde la perspectiva de la psi- 
cología conductual. De este trabajo se ha tomado el cuestionario de miedos dentales (CMD20. Pál-Hegedüs y Lejarza, 1992) como base para la construcción del cuestionario utilizado en la presente investigación.

4. González M, Ezpeleta I, Heredia M, Malpartida Z, Nuñez L, Trepiana R. Reacción de ansiedad de los hombres y mujeres al tratamiento dental. Psiquis 1999;20:111-4.

5. Locker D, Liddel A, Shapiro D. Diagnostic categories of dental anxiety; a porpulation-based study. Behav Res Ther 1999;37:25-37.

6. Liddell A, Locker D. Changes in levels of dental anxiety as a function of dental experience. Behav Mod 2000;24:57-68.

7. Schwarz E. Dental anxiety in Danish and Chinese adults-a cross- cultural perspective. Soc Sc Med 1995;41:123-30.

8. Sánchez A, Fernández JM, Iglesias C, González JC. Factores etiopatogénicos. En: Bobes J, Bousoño M, González MP, Sáiz PA, eds. Trastornos de Ansiedad y Trastornos Depresivos en Atención Primaria. Barcelona: Masson, 2001;17-36.

9. García E, Gil J, Rodríguez G. Análisis factorial. Madrid: La Muralla, 2000.

10. Sáiz PA, Durán A, Paredes B, Bousoño M. Manifestaciones clínicas de los trastornos de ansiedad. En: Bobes J, Bousoño M, González MP, Sáiz PA, eds. Trastornos de Ansiedad y Trastor- nos Depresivos en Atención Primaria. Barcelona: Masson, 2001;61-91.

11. Fernández C, Martín M, Tobal F. Miedo, ansiedad y fobia dental: definición, prevalencia y antecedentes. Ansiedad y Estrés 1995; 1:93-104.

12. Enrile de Rojas FJ, Buitrago-Vera PJ, CastañoSéiquer A, Sicilia-Felechosa A. Motivación de los pacientes en la consulta dental. RCOE 2000;5:535-42.

13. De Jongh A, Bongaarts G, Vermeule I, Visser K, De Vos P, Makkes P. Blood-injury-injection phobia and dental phobia. Behv Res Ther 1998;36:971-82.

14. Jensen B, Schroder U, Mansson U. Rectal sedation with diazepam or midazolam during extractions of traumatized primary incisors: A prospective, randomized, double blind trial in Swedish children aged $1.5-3.5$ years. Acta Odontol Scandi 1999;57:190-4.

15. Kaufman E, Davidson E, Sheinkmen Z,Magora $\mathrm{F}$, Lieblich S. Comparison between intranasal and intravenous midazolam sedation (with or without patient control) in a dental phobia clinic. J Oral Maxillofac Surg 1994;52:840-4.

16. Goodall E, File S, Sanders F, Skelly A. Self-ratings by phobic patients during dental treatment: greather improvement with nitrous oxide than midazolam. Human Psychopharmacology 1994;9:203-9.

17. Jöhren P, Jackowski J, Gängler P, Sartory G,
Thom A. Fear reduction in patients with dental treatment phobia. Bri J Oral Max Surg 2000;38:612-6

18. Goodall E, Skelly A, File S. Predictors of dental anxiety and effects of treatment with nitrous oxide and midazolam in dentally phobic patients. Hum Psychopharmacology 1994; 9:237-44.

19. De Jongh A, Muris P, Ter Horst G, Van Zuuren F, Schoenmakers N, Markker P. One-session cognitive treatment of dental phobia: preparing dental phobics for treatment by restructuring negative cognitions. Behav Res Ther 1995;33:947-54

20. De Jongh A, Muris P, Merckelbach H, Schoenmakers N. Suppression of dentist-related thoughts. Behavioural cognitive psychotherapy $1996 ; 24: 117-26$

21. Rodríguez M, Cano A. Relación entre adquisición y patrón de respuestas de ansiedad en la fobia dental. Ansiedad Estrés 1995;1:105-11.

22. De Jongh A, Muris P, Ter Horst G, Duyx M. Acquisition and maintance of dental anxiety: the role of conditioning experiences and cognitive factors. Behav Res Ther 1995;33:205-10.

23. Lehrner J, Eckersberger C, Walla P, Pötsch G, Deecke L. Ambient odor of orange in a dental office reduces anxiety and improves mood in female patients. Physiol Behav 2000; 71:83-6. 